\title{
BMJ Open Ventilatory function as a predictor of mortality in lifelong non-smokers: evidence from large British cohort studies
}

Ramyani P Gupta, David P Strachan

To cite: Gupta RP, Strachan DP. Ventilatory function as a predictor of mortality in lifelong non-smokers: evidence from large British cohort studies. BMJ Open 2017;7:e015381. doi:10.1136/ bmjopen-2016-015381

- Prepublication history and additional material are available. To view these files please visit the journal online (http://dx.doi org/10.1136/bmjopen-2016015381).

Received 30 November 2016 Revised 12 April 2017 Accepted 8 May 2017

CrossMark

Population Health Research Institute, St George's, University of London, London, UK

Correspondence to Professor David P Strachan; d. strachan@sgul.ac.uk

\section{ABSTRACT}

Background Reduced ventilatory function is an established predictor of all-cause mortality in general population cohorts. We sought to verify this in lifelong nonsmokers, among whom confounding by active smoking can be excluded, and investigate associations with circulatory and cancer deaths.

Methods In UK Biobank, among 149343 white neversmokers aged 40-69 years at entry, 2401 deaths occurred over a mean of 6.5-year follow-up. In the Health Surveys for England (HSE) 1995, 1996, 2001 and Scottish Health Surveys (SHS) 1998 and 2003 combined, there were 500 deaths among 6579 white never-smokers aged 40-69 years at entry, followed for a mean of 13.9 years. SD (z) scores for forced expiratory volume in the first second (FEV1) and forced vital capacity (FVC) were derived using Global Lung Initiative 2012 reference equations. These z-scores were related to deaths from all causes, circulatory disease and cancers using proportional hazards models adjusted for age, sex, height, socioeconomic status, region and survey.

Results In the HSE-SHS data set, decreasing z-scores for FEV1 (ZFEV1) and FVC (ZFVC) were each associated to a similar degree with increased all-cause mortality (hazard ratios per unit decrement $1.17,95 \% \mathrm{Cl} 1.09$ to 1.25 for zFEV1 and $1.19,95 \% \mathrm{Cl} 1.10$ to 1.28 for zFVC). This was replicated in Biobank (HRs 1.21, 95\% Cl 1.17 to 1.26 and $1.24,1.19$ to 1.29 , respectively). zFEV1 and zFVC were less strongly associated with mortality from circulatory diseases in HSE-SHS (HR 1.22, 95\% $\mathrm{Cl} 1.06$ to 1.40 for zFVC) than in Biobank (HR 1.47, 95\% Cl 1.35 to 1.60 for zFVC). For cancer mortality, HRs were more consistent between cohorts (for ZFVC: HRs $1.12,95 \% \mathrm{Cl} 1.01$ to 1.24 in HSE-SHS and 1.10, 1.05 to 1.15 in Biobank). The strongest associations were with respiratory mortality (for zFVC: HRs $1.61,95 \% \mathrm{Cl} 1.25$ to 2.08 in HSE-SHS and $2.15,1.77$ to 2.61 in Biobank).

Conclusions Spirometric indices predicted mortality more strongly than systolic blood pressure or body mass index, emphasising the importance of promoting lung health in the general population, even among lifelong non-smokers.

\section{INTRODUCTION}

Four decades of epidemiological research have consistently shown that reduced levels of ventilatory function, measured as one-second

\section{Strengths and limitations of this study}

- UK Biobank offers a spirometric study of lifelong non-smokers of unprecedented size, but the low participation rate may have compromised its generalisability.

- Assembling data from five national health surveys of England or Scotland produced a cohort of neversmokers, larger than in previously published studies, in which the generalisability of Biobank results could be tested.

- Within Biobank, the large numbers permitted statistically powerful subgroup analyses by sex, age, obesity and pre-existing disease. These within-cohort comparisons provide further reassurance about generalisability of associations between reduced ventilatory function and mortality.

- Mortality associations among the subset of Biobank participants whose spirograms met internationally recommended criteria for acceptability and reproducibility were very similar to the results among the full Biobank cohort, suggesting that the key findings are robust to inclusion or exclusion of participants with suboptimal spirometry.

- In common with previous studies of this issue, this analysis was restricted to fatal outcomes and therefore cannot distinguish between an association of reduced ventilatory function with disease incidence and an influence on case-fatality.

forced expiratory volume (FEV1) or forced vital capacity (FVC), are associated with higher all-cause mortality rates, and therefore shorter survival in the general population. ${ }^{1-7}$ Few studies have reported specifically on lifelong non-smokers, ${ }^{34}$ a group which form the minority of most populations surveyed hitherto, but are set to become more common in future as smoking becomes less prevalent in higher income countries.

Most publications have focused on FEV1, but a recent analysis ${ }^{7}$ of asymptomatic participants in the multiethnic Atherosclerosis Risk in Communities (ARIC) Study reported that all-cause mortality was strongly associated 
with diminished FVC, after adjustment for FEV1, but not the other way around, and there was no association between survival and the ratio of FEV1 to FVC. This conclusion was based on a combined analysis of smokers and non-smokers.

In this report, we compare the relationship of lung function measures (FEV1 and FVC) with subsequent mortality in UK Biobank (UKB) and in the Health Surveys for England (HSE) and Scottish Health Surveys (SHS). Biobank is the largest spirometric study ever performed in the UK and included a relatively high proportion of never-smokers. The national health surveys, although based on smaller numbers of subjects, recruited a wider age range, have a longer period of follow-up, higher response rates and are more representative of the general UK population than Biobank. The two sources are therefore complementary, in terms of precision and generalisability.

This paper focuses on findings for white lifelong non-smokers, among whom confounding by frequency or duration of active smoking can be excluded. We investigate associations of spirometric indices with total mortality and with major groups of causes of death, compare FEV1 and FVC as independent predictors of all-cause mortality and evaluate the possibility of reverse causation. We also compare results from UKB with those from the national health survey participants of a similar age at spirometric examination, to establish how widely generalisable are the findings from Biobank. Corresponding results for white former smokers and current smokers are included in the online supplement for completeness and described briefly in the text.

\section{METHODS}

\section{HSE and SHS}

Data were combined from the HSE 1995, 1996 and $2001^{8-10}$ and the SHS 1998 and $2003,{ }^{11-12}$ the years when spirometry was included in the protocol. These surveys aimed to recruit a representative sample of British adults through household sampling within selected parliamentary constituencies throughout England or Scotland. Participants were visited at home. Response rates ranged from $60 \%$ to $76 \%$ across the five surveys. The proportion of those visited who performed usable spirometry ranged from $63 \%$ to $84 \%$.

Spirometry was performed using handheld pneumotachograph spirometers (Vitalograph Escort) with the best results of FEV1 and FVC recorded from three technically satisfactory blows. No flow-volume curves or reproducibility criteria were available for assessment. Valid lung function measurements were available for 6579 lifelong non-smokers aged 40-69 years and 1429 aged 70 years or more at the start of follow-up, all of white ethnicity.

Deaths occurring up to April 2013 were available for analysis in the HSE, ${ }^{13}$ and deaths up to December 2011 were linked in the SHS. ${ }^{14}$ Combining all five surveys, there were 500 deaths among white never-smokers aged
40-69 years at recruitment over a mean follow-up period of 13.9 years. Deaths from respiratory disease, circulatory disease, cancer and all other causes were coded using International Classification of Diseases (ICD9) (460-519, 390-459, 140-208 and all others, respectively) and ICD10 (chapters J, I, C and all others, respectively).

Smoking history was self reported. Socioeconomic status was measured at the level of the household, based on the social class of the head of the household. Nation (England or Scotland), region (within England) and survey year were included as additional covariates.

\section{UK Biobank}

This study recruited 502682 volunteers aged $40-69$ years in 22 recruitment centres throughout England, Wales and Scotland during 2006-2010, following invitations to 9 million people. ${ }^{15}$ Spirometry was performed using a handheld pneumotachograph spirometer (Pneumotrac 6800 ) from which volume-time arrays were stored for each blow. ${ }^{16}$

Eighty-one per cent of the cohort performed two blows with acceptable start and measures of FEV1 reproducible within $250 \mathrm{~mL}$. This was considered the most inclusive sample of 'usable spirograms'. When end-blow quality was also considered, $58 \%$ of the cohort had evidence of a good plateau and both FEV1 and FVC reproducible within $150 \mathrm{~mL}$, the criteria recommended by the American Thoracic Society/European Respiratory Society (ATS/ ERS) Task Force on Standardisation of Spirometry. ${ }^{17}$ This subgroup of $58 \%$ was considered to be the best quality' spirograms, among which to evaluate the relative importance of FEV1 and FVC as predictors of mortality.

The present analysis is based on deaths occurring up to mid-August 2015, a mean follow-up period of 6.5 years. There were 2401 deaths among 149343 lifelong non-smokers aged 40-69 years of white ethnicity who performed 'usable spirograms. Deaths from respiratory disease, circulatory disease, cancer and all other causes were coded using ICD10 (chapters J, I, C and all others, respectively).

Smoking history was self reported. Socioeconomic status was measured at the level of residential area, using the Townsend Deprivation Index, grouped into quartiles for analysis. Biobank recruitment centre was used as an additional covariate to adjust for possible regional differences.

\section{Adjustment of spirometric measures for gender, age and height}

The Global Lung Initiative (GLI) 2012 reference equations for white ethnic groups ${ }^{18}$ were used in both sets of data to standardise FEV1 and FVC for age, sex and height. The GLI-2012 equations generate a 'z-score' which represents the relative position of an individual among the distribution predicted for lifelong non-smokers with no history of lung disease of the same gender, age and height. This allows for the spread of predicted values to differ by age, height and gender, expressing the relative ranking of an 
individual in terms of a SD (z) score. For each individual in the analysis, there were three GLI-2012 z-scores, corresponding to their relative ranking for FEV1 (zFEV1), FVC (zFVC) and the ratio FEV1:FVC (zFEVFVC). Outlying observations were excluded by restricting all the analyses in both data sets to values of zFEV1 and zFVC within the range -5 to +5 z-score units. This exclusion removed $0.2 \%$ of UKB participants, $0.5 \%$ of participants in the national health surveys aged $40-69$ years and $0.3 \%$ of national health survey participants aged 70 years or more.

\section{Modelling of mortality}

The relationship of spirometric indices with subsequent mortality was modelled by proportional hazards (Cox) regression, which estimates the relative increase in mortality rate (HR) for a unit change in each explanatory variable. The z-scores are expressed on a SD scale, so HRs for zFEV1 and zFVC are expressed per unit decrement (ie, an increase in risk for a decrease in lung function). A typical range of z-scores among lifelong non-smokers would be 4 units. A HR of 1.2 per unit decrement corresponds approximately to a twofold difference in mortality rate across this range.

Due to the high correlation between zFEV1 and zFVC among lifelong non-smokers (0.88 in Biobank, 0.80 in national health survey participants aged 40-69 years), we modelled the association of mortality with zFEV1 both alone and jointly with zFEVFVC, and similarly for zFVC. Among never-smokers, the correlations between zFEV1 and zFEVFVC (0.35 in Biobank, 0.36 in the national surveys) and between zFVC and zFEVFVC $(-0.13$ in Biobank, -0.20 in the national surveys) were weak enough to avoid major collinearity in the joint models. The significance of the HR for zFEVFVC when modelled jointly with $\mathrm{zFEV1}$ was used to assess whether $\mathrm{zFVC}$ predicted mortality independent of zFEV1, and vice versa when zFEVFVC was modelled jointly with zFEV1.

All proportional hazards models were restricted to white participants and adjusted for sex, age, standing height, socioeconomic status and region. Analyses of data from the five national health surveys were additionally adjusted for survey year as a categorical variable. Since each survey was conducted in a different year, inclusion of survey year in the model is closely equivalent to a fixed-effect meta-analysis of the results from each of the five surveys. In a more formal two-stage individual participant meta-analysis for all-cause mortality in lifelong non-smokers, there was no substantial or significant heterogeneity of HRs among the five national health surveys $\left(I^{2}=0.0 \%, p=0.775\right.$ for $z F E V 1 ; I^{2}=10.5 \%, p=0.346$ for $\mathrm{zFVC}$ ). Therefore, for simplicity of presentation we report results for the five national surveys combined, but analyse UKB separately because one of our objectives is to investigate how closely these two sets of results correspond. Heterogeneity between HRs for the pooled national surveys and UKB was assessed by testing the significance of the difference between the corresponding $\log$-HRs from these two data sets.
The assumption of proportionality of hazards was assessed by log-log plots and by fitting zFEV1 or zFVC as a time-dependent covariate in the model. No strong or statistically significant evidence of time dependence emerged for all-cause mortality among lifelong non-smokers. Nevertheless, results for all-cause mortality were partitioned at 5 years of follow-up for two reasons. First, because the minimum duration of follow-up in UKB was 4.87 years, so virtually all of that cohort had been followed for 5 years or more, allowing a more direct comparison with results from the national health surveys, all of which had been followed for more than 5 years. The second reason for partitioning at 5 years was to address the possibility of reverse causation (impaired spirometric performance due to pre-existing conditions which lead to early death). Reverse causation was also investigated by analysing mortality in subgroups with no prior history of respiratory disease, circulatory disease or cancer.

\section{RESULTS}

\section{Participant characteristics}

Online supplementary e-table 1 summarises the number of participants, duration of follow-up and deaths from all causes and subgroups of cause, in each data set, by sex, age and smoking history.

\section{All-cause mortality}

Table 1 compares the HRs for age-sex-height-adjusted FEV1 and FVC in relation to all-cause mortality in the combined HSE and SHS data set, and UKB, among participants aged 40-69 years at entry. All 'usable spirograms' from Biobank were included in this comparison, because no additional quality control had been applied in the national health surveys 1995-2003.

When each spirometric index was modelled singly, all associations were highly statistically significant $(p<0.0001)$. Among lifelong non-smokers in both cohorts, FEV1 and FVC displayed similar strengths of association with all-cause mortality (table 1 ). Among former smokers, FEV1 was the stronger predictor in HSE-SHS but FEV1 and FVC showed similar strength of association with all-cause mortality in UKB. Among current smokers in both data sets, FEV1 was a stronger predictor of all-cause mortality than FVC (see online supplementary e-tables 2-4).

Table 1 also shows the most direct comparison between the two data sets, based on all-cause mortality within 5 years. Although the associations of spirometric indices with these earlier deaths were stronger in HSE-SHS than in UKB, the differences between the cohorts were not statistically significant ( $p=0.23$ for FEV1, $p=0.39$ for $F V C$ ). Associations with deaths after 5 years are less comparable between the data sets, due to the shorter period of follow-up in UKB.

Stronger associations of FEV1 and FVC with earlier deaths than with later mortality from all causes were also evident among former smokers and current smokers, 


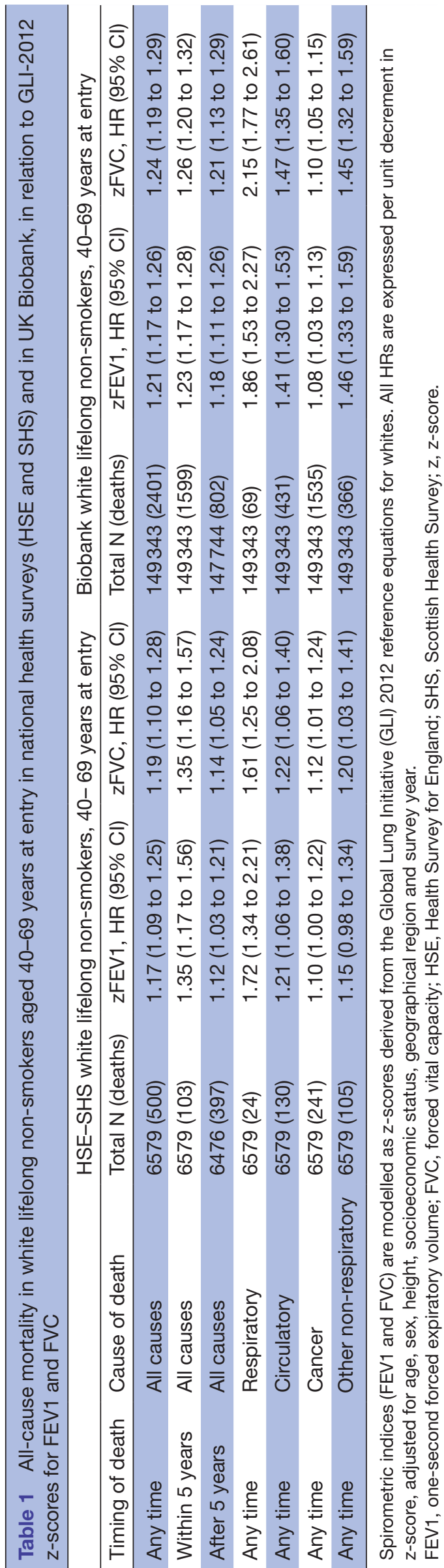

although the differences were more marked in HSE-SHS than in UKB (see online online supplementary e-tables 1 and 3). Formal tests for time dependence of the HR for all-cause mortality found statistically significant reduction in HR with increasing follow-up time $(\mathrm{t})$ only among ex-smokers $(\mathrm{p}=0.00032$ for $\mathrm{zFEV} 1 * \mathrm{t}$ in UKB, $\mathrm{p}=0.021$ for zFEV1*t in HSE-SHS, $\mathrm{p}=0.000006$ for $\mathrm{zFVC} * \mathrm{t}$ in UKB, $\mathrm{p}=0.001$ for $\mathrm{zFVC} * \mathrm{t}$ in HSE-SHS). Among never-smokers and current smokers in both data sets, the reduction in HR with increasing follow-up was small (a relative reduction of $1 \%$ per year) and non-significant $(p>0.05)$ for both FEV1 and FVC.

These analyses confirm that the modelling assumption of proportionality of hazards over the duration of follow-up is valid, at least for lifelong non-smokers, in both data sets.

\section{Influence of spirogram quality}

Table 2 presents the results for all causes of death among Biobank participants with 'best quality' spirograms, for comparison with those obtained from the full set of 'usable spirograms'. The pattern and magnitude of the results among the former subset are very similar to the overall UKB results. The covariate-adjusted HRs for all-cause mortality comparing the 'best quality' group to the remainder were: HR 0.94 (95\% CI 0.86 to 1.02$)$ among lifelong non-smokers, 1.02 (0.96 to 1.09) among former smokers and 0.94 (0.85 to 1.04) among current smokers. None of these HRs are statistically significant, despite very large number of subjects included each comparison.

\section{Choice of spirometric index}

Among lifelong non-smokers, adding FEV1:FVC ratio to a model including FVC did not contribute additional information, whereas adding FEV1:FVC ratio to a model including FEV1 did improve the fit of the model significantly (table 2). This pattern was evident in both HSE-SHS and UKB, and among the subset of UKB participants with 'best quality' spirograms.

\section{Cause-specific mortality}

Table 1 also presents the association of spirometric indices (modelled singly) with respiratory, circulatory, cancer and other causes of death among lifelong non-smokers. The strength of association with FEV1 and FVC was greatest for respiratory mortality and weakest for cancer deaths. This applied in both data sets, but the HRs for respiratory, circulatory and other causes of death were greater in UKB than in HSE-SHS. The results in the two cohorts are more similar for cancer mortality. The heterogeneity of HRs between the two data sets was statistically significant only for FVC in relation to circulatory mortality $(\mathrm{p}=0.025)$, and for both FEV1 $(\mathrm{p}=0.009)$ and FVC $(\mathrm{p}=0.042)$ in relation to causes of death other than respiratory, circulatory or cancer among never-smokers. Within UKB, results for cause-specific mortality were generally consistent between the 'best spirogram' subgroup and the fuller 


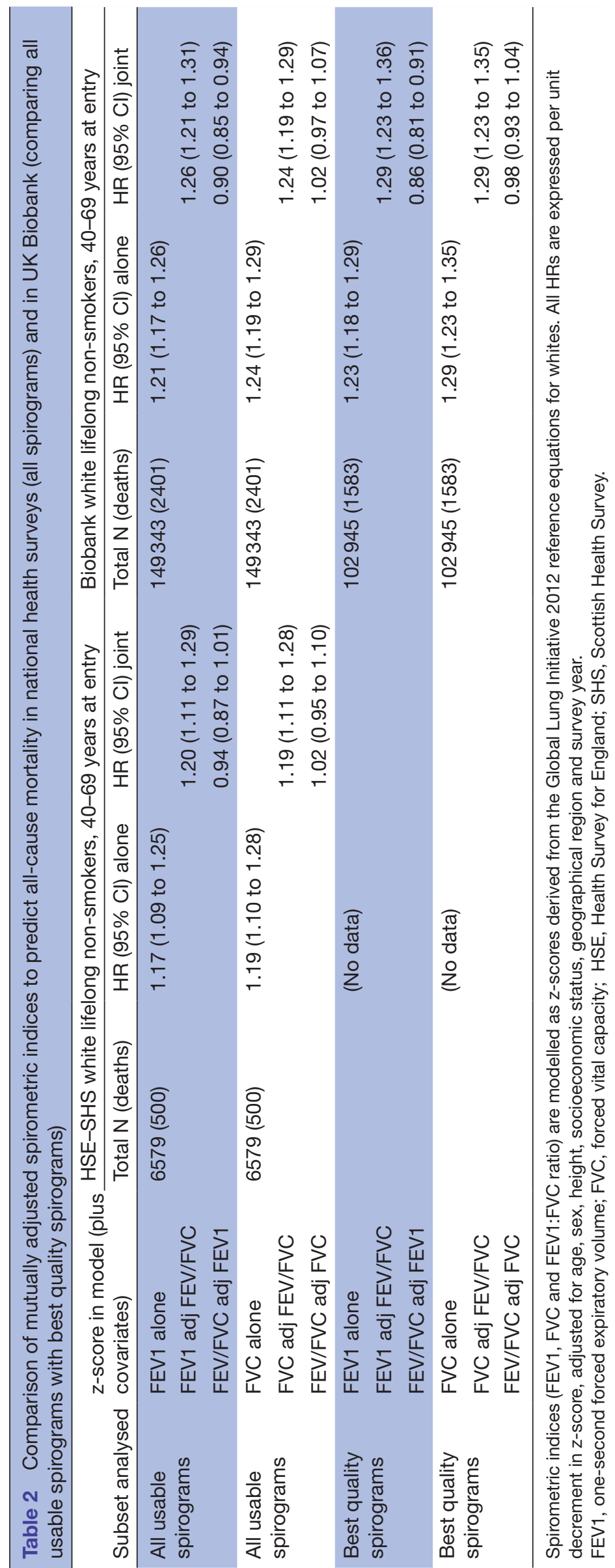

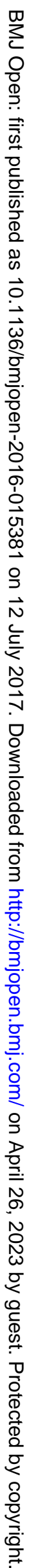


data set in all smoking subgroups (see online supplementary e-table 4).

\section{Comparisons by subgroups of age and sex}

Online supplementary e-table 3 shows that within UKB the general pattern of results was similar in both sexes. There was no statistically significant effect modification by sex for either spirometric index in any smoking subgroup ( $p \geq 0.10$ for each interaction test).

Online supplementary e-table 3 also compares the results for all-cause mortality among younger (aged 40-59 years) and older (aged 60-69 years) UKB participants. The HRs in all smoking groups were consistent between these two age subgroups and there were no statistically significant age interactions for either spirometric index $(\mathrm{p}>0.40)$.

Online supplementary e-table 5 compares the spirometric associations with all-cause and cause-specific mortality among HSE and SHS participants aged 40-69 years and 70 years or more at entry. The pattern for all causes was consistent between these age groups in never-smokers and current smokers $(p>0.2)$, but there were significant age interactions among ex-smokers $(\mathrm{p}<0.006)$.

Online supplementary e-table 3 compares the results for all-cause mortality among obese and non-obese Biobank participants. Although the pattern of results was generally consistent between these categories in all smoking subgroups, a statistically significant difference in the HR for FVC occurred among current smokers $(p=0.022)$. Other interactions with obesity were non-significant $(\mathrm{p} \geq 0.10)$.

\section{Comparisons by prior disease history in UKB}

Table 3 compares the spirometric associations with all-cause mortality in Biobank participants with and without a history of respiratory disease at the baseline spirometric examination. In the 18545 (12\%) white never-smokers with 'usable spirograms' who had a history of diagnosed respiratory disease (of which $90 \%$ reported asthma), FVC was a stronger predictor of all-cause mortality than FEV1. Among the subgroup without a respiratory history, results were similar to the full cohort.

Lung function also emerged as a significant predictor of circulatory mortality among those with and without a prior history of heart attack, angina, stroke, thrombosis or hypertension (table 3 ). This pattern was confirmed among former smokers and current smokers (see online supplementary e-table 3 ).

Finally, the association of FEV1 and FVC with cancer mortality was shown to be stronger in those with a prior cancer diagnosis (table 3), but it was not statistically significant, despite the large sample size, among those with no cancer history at the spirometric examination. However, this finding is confined to the lifelong non-smokers: both FEV1 and FVC were more strongly and significantly associated with cancer death among former smokers and current smokers with no cancer history (see online supplementary e-table 6). Cancer mortality among participants with a history of cancer at entry was strongly and significantly associated with both FEV1 and FVC in all three smoking subgroups (see online supplementary e-table 6).

\section{Comparison of spirometry with other predictors of mortality}

Figure 1 (data in online supplementary e-table 7) compares the relative mortality across quartiles of body mass index, systolic blood pressure and FVC z-score, for all deaths and for deaths from circulatory disease, among white lifelong non-smokers in HSE-SHS and in UKB. A similar pattern emerged for all-cause mortality in both cohorts, with differentials in mortality across quartiles of zFVC being at least as great as those across quartiles of body mass index or systolic blood pressure.

\section{DISCUSSION \\ Principal findings}

A broadly coherent picture emerges from this comparison of UK national cohorts. Ventilatory function, even if measured imperfectly, consistently predicted both respiratory deaths and non-respiratory mortality from a range of causes. This was found even among lifelong non-smokers, so confounding by the amount or duration of active smoking is not the sole explanation. Both for all-cause mortality and more specifically for circulatory disease mortality, FEV1 and FVC were as strongly predictive as body mass index and more strongly predictive than systolic blood pressure.

\section{Strengths and weaknesses of this study}

UKB offers a spirometric study of lifelong non-smokers of unprecedented size, but its $5.5 \%$ participation rate may have compromised its generalisability. Assembling data from five UK national health surveys produced a cohort of never-smokers, larger than the combined number of participants in previous publications ${ }^{3,4}$ in which the generalisability of Biobank results could be tested. The similar pattern of results in HSE-SHS and UKB suggests that the key findings are generalisable, at least to the British population.

Within Biobank, the large numbers permitted subgroup analyses by sex, age, obesity and pre-existing disease, of sufficient statistical power to exclude important interaction effects. These within-cohort comparisons provide further reassurance about the generalisability of the principal findings among lifelong non-smokers.

Although only $58 \%$ of the Biobank cohort performed spirometry which fulfilled internationally recommended criteria for acceptability and reproducibility, ${ }^{17}$ the results in this subgroup were very similar to those among the full set of Biobank participants who performed 'usable' spirometry. Those results were, in turn, consistent with the findings from national health surveys where the acceptability and reproducibility of spirometry was not formally assessed in the field. These within-cohort and cross-cohort comparisons suggest that the principal 
findings are robust to inclusion or exclusion of participants with suboptimal spirometric performance.

In common with previous studies of this topic, our analysis was restricted to fatal outcomes and therefore cannot distinguish between an influence of reduced ventilatory function on disease incidence and an effect on case fatality. The association with cancer mortality was weaker among those with no cancer diagnosis at entry, suggesting an association primarily with case fatality. In contrast, the association of spirometric indices with circulatory mortality was equally strong in those with and without a prior history of circulatory disease. The recent linkage of hospital admissions and primary care consultations to the UKB cohort will allow associations with incidence and case fatality to be investigated more directly in future.

\section{Comparison with other studies}

Previous studies of lifelong non-smokers have been of limited size: 662 males and 2048 females in the Copenhagen City Heart Study, of whom 195 died during 10 years of follow-up, ${ }^{3}$ and 3562 male London civil servants in the Whitehall Study, of whom 408 died over a period of 18 years. ${ }^{4}$ The Whitehall cohort was subsequently followed for 33-35 years, accumulating 1545 deaths among 3083 lifelong non-smokers. ${ }^{19}$ In the analyses we present here, there are almost twice this number of deaths among lifelong non-smokers, despite a shorter follow-up period, due to the much larger sample size at entry, particularly in UKB.

The two publications from the Whitehall cohort ${ }^{4,19}$ compared FEV1 and height as predictors of all-cause and cause-specific mortality among never-smokers. The first ${ }^{4}$ found that FEV1 predicted mortality independent of height, but height did not predict survival independent of FEV1. The second ${ }^{19}$ found that FEV1 and height were similarly related both to mortality and to a range of other risk factors, concluding that both FEV1 and height may be markers of early life exposures of relevance to longevity.

Published analyses of the Whitehall cohort ${ }^{4,19}$ assessed only FEV1, but not FVC. In the Copenhagen study, ${ }^{3}$ the association of all-cause mortality among never-smokers was slightly stronger and more statistically significant with FVC than with FEV1 (both spirometric indices analysed as per cent predicted for age, sex and height). However, no formal comparison was made between the mortality risks associated with the two indices. An analysis of mortality over an average follow-up period of 13.7 years among 7489 participants aged 45-64 years in the US ARIC cohort ${ }^{7}$ suggested that FVC should be considered as a more predictive spirometric index than FEV1, but this conclusion was drawn from a cohort of mixed smoking habits.

In our study of lifelong non-smokers, we confirmed that FVC (rather than FEV1) is the index of greater importance in determining survival in middle-aged never-smokers. In contrast, among current smokers, FEV1 emerged as the more influential predictor. This may be because the 

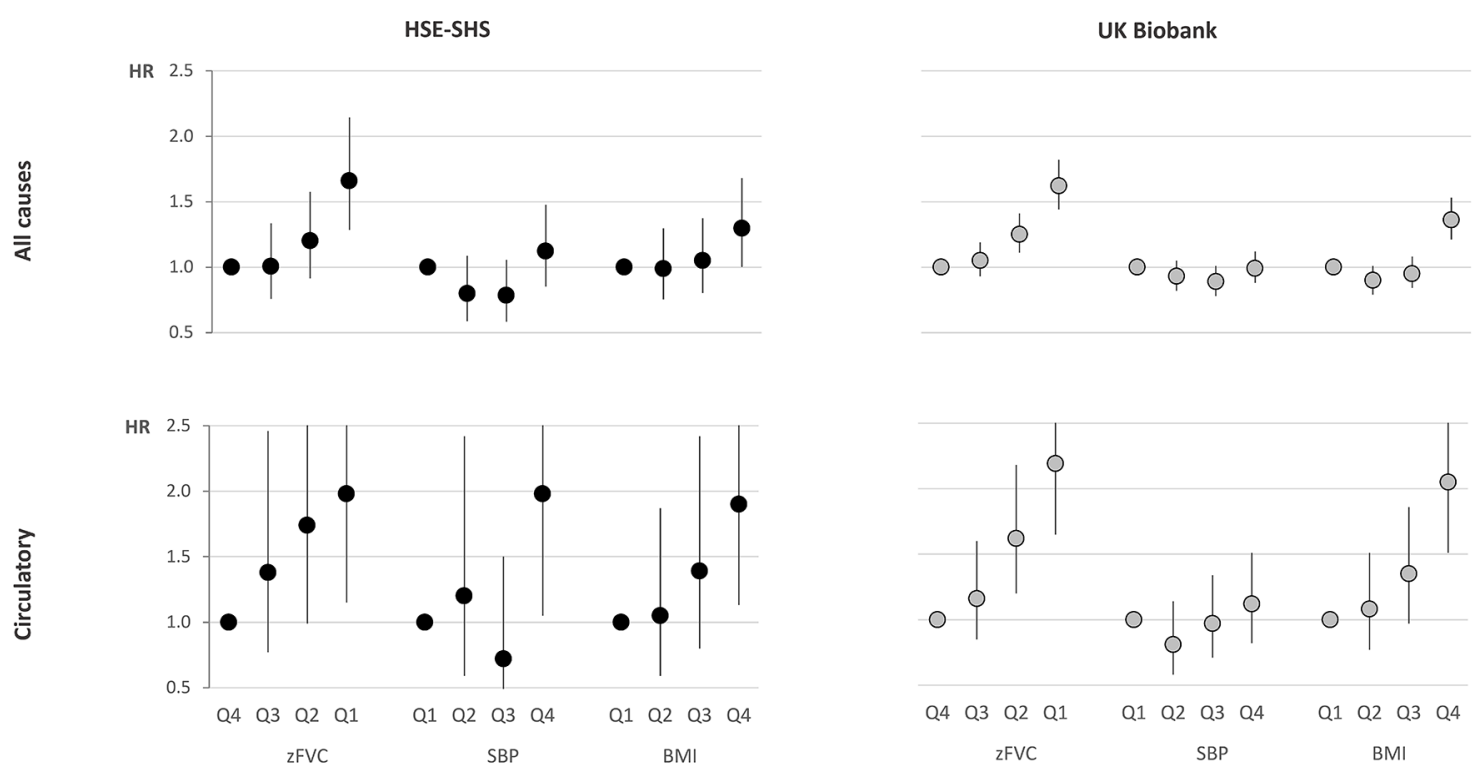

Figure 1 Hazard ratios for death from all causes, and from circulatory diseases, by quartile of age-sex-height-adjusted zFVC, SBP and BMI among white lifelong non-smokers aged 40-69 years at entry in national health surveys (HSE and SHS) and in UK Biobank. HRs are adjusted for age, sex, height, socioeconomic status, region and survey year. The reference category $(H R=1)$ is the highest quartile (Q4) for ZFVC and the lowest quartile (Q1) for SBP and BMI. Whiskers represent the 95\% Cl for each HR. Data included in online supplementary e-table 7. BMI, body mass index; FVC, forced vital capacity; HSE, Health Survey for England; SBP, systolic blood pressure; SHS, Scottish Health Survey; z, z-score.

FEV1:FVC ratio declines with both the dose and duration of smoking, and these also increase mortality risk.

The ability to perform good quality spirometry is an integrated assessment of physical and cognitive function and therefore might be considered a predictor of mortality in its own right. In the US Six Cities Study, excessively variable spirometric performance was an indicator of poor health and associated with shorter survival..$^{20}$ In contrast, the mortality experience of Biobank participants who produced 'best quality' spirograms did not differ greatly from that of their peers who produced "'usable' 'but not' 'best quality' blows.

\section{Possible implications}

In clinical practice, particularly in primary care, quality control of spirometry is unlikely to be much better than in the national health surveys where lung function was tested by a trained research nurse in the home setting. Therefore, while the results from the Biobank 'best quality' subgroup are of confirmatory interest, the more inclusive results for all 'usable spirograms' may be more generally relevant.

In both cohorts, age-sex-height-adjusted lung function emerged as a stronger predictor of all-cause mortality than either systolic blood pressure or body mass index, which are, respectively, the second and sixth most influential causes worldwide of loss of healthy lifespan, as measured by disability-adjusted life years. ${ }^{21}$ It is therefore puzzling to find $\mathrm{U}$-shaped or J-shaped relationships of mortality with these two cardiovascular risk factors, but the similar patterns of results in Biobank and the national health surveys suggest that this is not a unique feature of either of these British cohorts.

Specifically for circulatory disease mortality, FEV1 and FVC were as strongly predictive as body mass index and more strongly predictive than systolic blood pressure. Therefore, spirometry may deserve consideration as an addition to cardiovascular risk scoring algorithms in future.

\section{CONCLUSION}

More generally, these results emphasise the potential importance of promoting and protecting lung health in the general population, even among lifelong non-smokers with no history of respiratory disease.

Acknowledgements This research has been conducted using the UK Biobank resource (application no 412) and national health surveys data obtained from the UK Data Archive.

Contributors The study was conceived by DPS. Design and analysis of the Health Surveys for England and Scottish Health Surveys modelling was conducted by RPG. Design and analysis of the UK Biobank modelling was conducted by DPS. Both authors contributed to interpretation of the findings and writing of the manuscript.

Funding The analyses presented here were supported by a project grant from the British Lung Foundation (ref: RHotN12-14). Neither UK Biobank nor the UK Data Archive nor the British Lung Foundation have been involved in the writing of the manuscript.

Competing interests None declared.

Ethics approval This is a secondary analysis of anonymised data from national health surveys, each of which obtained ethics committee approval for their fieldwork, ${ }_{8-12,15}$ but no specific ethical approval was required for this data analysis.

Provenance and peer review Not commissioned; externally peer reviewed. 
Data sharing statement The corresponding author DPS has full access to all the data included in theseanalyses and is the guarantor of this manuscript.

Open Access This is an Open Access article distributed in accordance with the Creative Commons Attribution Non Commercial (CC BY-NC 4.0) license, which permits others to distribute, remix, adapt, build upon this work non-commercially, and license their derivative works on different terms, provided the original work is properly cited and the use is non-commercial. See: http://creativecommons.org/ licenses/by-nc/4.0/

(c) Article author(s) (or their employer(s) unless otherwise stated in the text of the article) 2017. All rights reserved. No commercial use is permitted unless otherwise expressly granted.

\section{REFERENCES}

1. Ashley F, Kannel WB, Sorlie PD, et al. Pulmonary function: relation to aging, cigarette habit, and mortality. Ann Intern Med 1975;82:739-45.

2. Beaty $\mathrm{TH}$, Cohen BH, Newill CA, et al. Impaired pulmonary function as a risk factor for mortality. Am J Epidemiol 1982;116:102-13.

3. Lange P, Nyboe J, Appleyard M, et al. Spirometric findings and mortality in never-smokers. J Clin Epidemiol 1990;43:867-73.

4. Strachan DP. Ventilatory function, height, and mortality among lifelong non-smokers. J Epidemiol Community Health 1992;46:66-70.

5. Schünemann HJ, Dorn J, Grant BJ, et al. Pulmonary function is a long-term predictor of mortality in the general population: 29-year follow-up of the Buffalo Health Study. Chest 2000;118:656-64.

6. Mannino DM, Buist AS, Petty TL, et al. Lung function and mortality in the United States: data from the First National Health and Nutrition Examination Survey follow up study. Thorax 2003;58:388-93.

7. Burney PG, Hooper R. Forced vital capacity, airway obstruction and survival in a general population sample from the USA. Thorax 2011;66:49-54.

8. Joint Health Surveys Unit of Social and Community Planning Research and University College London. Health Survey for England, 1995. 4th ed: UK Data Service, 2010. data collection. http://dx.doi. org/10.5255/UKDA-SN-3796-1 (accessed 5 Oct 2016).

9. Joint Health Surveys Unit of Social and Community Planning Research and University College London. Health Survey for England, 1996. 4th ed: UK Data Service, 2010. data collection. http://dx.doi. org/10.5255/UKDA-SN-3886-1 (accessed 5 Oct 2016).
10. National Centre for Social Research, University College London. Department of Epidemiology and Public Health. Health Survey for England, 2001. 3rd ed: UK Data Service, 2010. data collection. http:// dx.doi.org/10.5255/UKDA-SN-4628-1 (accessed 5 Oct 2016).

11. Joint Health Surveys Unit of Social and Community Planning Research and University College London. Scottish Health Survey, 1998. UK Data Service, 2001. data collection. http://dx.doi.org/10. 5255/UKDA-SN-4379-1 (accessed 5 Oct 2016).

12. Joint Health Surveys Unit, University College London. Scottish Health Survey, 2003. 2nd ed: UK Data Service, 2011. data collection. http://dx.doi.org/10.5255/UKDA-SN-5318-1 (accessed 5 Oct 2016).

13. Mindell J, Biddulph JP, Hirani V, et al. Cohort profile: the health survey for England. Int J Epidemiol 2012;41:1585-93.

14. Gray L, Batty GD, Craig P, et al. Cohort profile: the scottish health surveys cohort: linkage of study participants to routinely collected records for mortality, hospital discharge, cancer and offspring birth characteristics in three nationwide studies. Int J Epidemiol 2010;39:345-50.

15. Sudlow C, Gallacher J, Allen N, et al. UK biobank: an open access resource for identifying the causes of a wide range of complex diseases of middle and old age. PLoS Med 2015;12:e1001779.

16. UK Biobank: Protocol for a large-scale prospective epidemiological resource. Protocol No: UKBB-PROT-09-06 (Main Phase). UK Biobank Coordinating Centre, 2007. http://www.ukbiobank.ac.uk/wp-content/ uploads/2011/11/UK-Biobank-Protocol.pdf (accessed 6 Oct 2016).

17. Miller MR, Crapo R, Hankinson J, et al. ATS/ERS Task Force. General considerations for lung function testing. Eur Respir $J$ 2005;26:153-61.

18. Quanjer PH, Stanojevic S, Cole TJ, et al. Multi-ethnic reference values for spirometry for the 3-95-yr age range: the global lung function 2012 equations. Eur Respir J 2012;40:1324-43.

19. Batty GD, Gunnell D, Langenberg C, et al. Adult height and lung function as markers of life course exposures: associations with risk factors and cause-specific mortality. Eur J Epidemiol 2006;21:795-801.

20. Eisen EA, Dockery DW, Speizer FE, et al. The association between health status and the performance of excessively variable spirometry tests in a population-based study in six U.S. cities. Am Rev Respir Dis 1987;136:1371-6.

21. Forouzanfar MH, Alexander L, Anderson HR, et al. Global, regional, and national comparative risk assessment of 79 behavioural, environmental and occupational, and metabolic risks or clusters of risks in 188 countries, 1990-2013: a systematic analysis for the Global Burden of Disease Study 2013. Lancet 2015;386:2287-323. 